\title{
Effect of Anesthesia on Fetal-Maternal Heart Rate Variability and Coupling in Pregnant Mice and Fetuses
}

\author{
Ahsan H. Khandoker ${ }^{1}$, Maisam Wahbah ${ }^{1}$, Chihiro Yoshida $^{2}$, Yoshitaka Kimura ${ }^{2}$, Yoshiyuki \\ Kasahara $^{2}$ \\ ${ }^{1}$ Khalifa University, Abu Dhabi, UAE \\ 2 Tohoku University, Sendai, Japan
}

\begin{abstract}
To evaluate and assess the cardiovascular system during fetal development in the utero of pregnant mouse, it is essential to understand the effect of mandatory anesthesia treatment on sympathetic and parasympathetic nervous system activities. The preliminary study presented in this paper explores the changes in fetal and maternal Heart Rate Variability (HRV) parameters as well as fetalmaternal Heart Rate (HR) coupling measures during anesthesia. ECG signals of 6 pregnant mice and 10 fetuses were recorded for $15 \mathrm{~min}$. The obtained ECG signals were segmented into three periods, each for a duration of $5 \mathrm{~min}$. Maternal and fetal HRV parameters in addition to fetalmaternal coupling patterns were computed for each of the three segments of the ECG signals. During the first $10 \mathrm{~min}$, results show that mean and root mean square of successive differences (RMSSD) of maternal HR did not change, but significantly decreased after the first $10 \mathrm{~min}$. A similar result was observed for the mean, RMSSD and standard deviation of NN intervals in fetal HR. On the other hand, no significant changes were observed for the coupling patterns between fetal-maternal heartbeats. These observations suggest that fetal nervous system activities were suppressed by anesthesia treatment applied to pregnant mice for more than $10 \mathrm{~min}$.
\end{abstract}

\section{Introduction}

Anesthesia is essential in any surgical procedure. This treatment is of our particular interest during newborn delivery. There is still little knowledge of how anesthesia affects the heart rates of mother and her fetus when considering fetal development.

Fetal mice models have been frequently used to study human congenital diseases $[1,2]$. In addition to that, such models have been commonly used to explore maternal factors influences on fetal development with the aid of drug manipulations $[3,4]$. The advantage of using mouse mod- els in particular is their fast development, in which the fetus requires only three weeks to fully grow [5]. This is of vital importance when conducting research activities concerning the assessment of fetal development in general, and the cardiovascular system in the utero of pregnant mouse in particular.

Beat-to-beat Heart Rate Variability (HRV) from Electrocardiography (ECG) signals provides a noninvasive way to monitor the activity of autonomic nervous system [6]. To study fetal HRV [7] in utero, anesthesia is required to immobilize mother mouse for surgical procedure. This goal calls for a dedicated study on fetal and maternal cardiovascular responses to anesthesia in pregnant mice, which has not yet been discussed in the literature.

The main objective of the preliminary study presented in this paper is to investigate the changes in fetal and maternal HRV parameters as well as fetal-maternal Heart Rate (HR) coupling measures during anesthesia. ECG signals of pregnant mice and their fetuses were simultaneously recorded for $15 \mathrm{~min}$. The obtained ECG signals were segmented into three periods: the first 5 min (P1), the second $5 \mathrm{~min}(\mathrm{P} 2)$, and the last $5 \mathrm{~min}(\mathrm{P} 3)$. Maternal and fetal HRV parameters [8], such as mean HR (MHR), standard deviation of NN intervals in HR (SDNNHR), and root mean square of successive differences between normal heartbeats (RMSSDHR) were computed for each of the three segments. Additionally, the relative phase and coupling strength between fetal and maternal heartbeats were estimated for all three segments.

\section{Methods}

\subsection{Mice subjects and experiment protocol}

The experiments conducted in this study followed the animal experimentation guidelines of Tohoku University in Japan. Additionally, the experimental protocol was approved by the Tohoku University Committee for Safety Management of Animals. A total of 10 fetuses from 
6 pregnant female mice of type C57BL/6J with body weight of 16-22 $\mathrm{g}$ were used in this study. Mice subjects were housed in cages under control lighting of $12: 12 \mathrm{~h}$ light-dark cycle and temperature of $22 \pm 2^{\circ} \mathrm{C}$. On day 17.5 of gestation, pregnant mice were anesthetized with subcutaneous ketamine (Ketalar $500 \mathrm{mg}$ Daiichi Sankyo: $100 \mathrm{mg} / \mathrm{kg}$ ) and xylazine (Rompun Injection Solution 2\% Bayer; $10 \mathrm{mg} / \mathrm{kg}$ ), and maintained with inhalational isoflurane (Forane AbbVie Inc. 0.5\%, $260 \mathrm{ml} / \mathrm{min}$ ). ECG signals of fetuses and pregnant mice were simultaneously recorded for 15 min using a recording system for embryonic mouse as explained in our previous study [9]. Fig. 1 shows the experimental setup along with examples of the recorded fetal and maternal ECG signals.
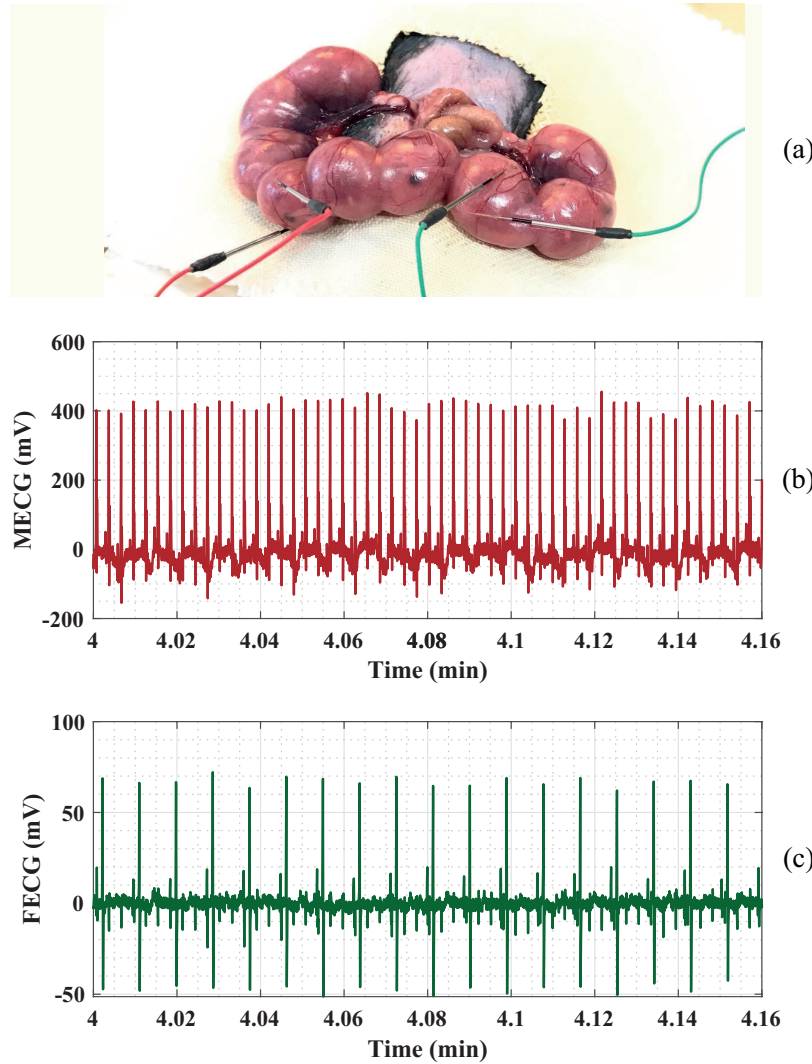

Figure 1. The panels in this figure include: (a) the experimental setup of the fetal ECG recording system, and examples of ECG signals for the (b) mother and (c) fetus.

\subsection{Heart Rate Variability}

The procedure to monitor and estimate beat-to-beat HR and its variability parameters to assess autonomic neurodevelopment in mouse fetuses -while in utero- by using fetal ECG approach was demonstrated in our previous study [7]. Fetal and Maternal Heart Rate Variability (FHRV and MHRV) features [8], including: the mean value of
HR (FMHR and MMHR), standard deviation of NN intervals in HR (FSDNNHR and MSDNNHR), and root mean square of successive differences between normal heartbeats (FRMSSDHR and MRMSSDHR), were estimated from three segments (P1 (0-5 min), P2 (5-10 min), and P3 (10-15 min)) of the recorded ECG signals.

\subsection{Coupling of Heartbeats}

The coupling/synchronization between R-peaks of fetal and maternal ECG signals was estimated by phase coherence method as described in [10]. The instantaneous phase time series was obtained by

$$
\varphi\left(t_{k}\right)=\frac{2 \pi\left(t-t_{k}\right)}{\left(t_{k+n}-t_{k}\right)}+2 \pi k
$$

where $t$ and $t_{k}$ are the times of R-peak of maternal and fetal ECG signals respectively, and $n$ is fetal heartbeats. The relative phase $\psi\left(t_{k}\right)$ in the time window of $t_{w}$ with respect to maternal ECG signal was calculated using the equation

$$
\psi\left(t_{k}\right)=\frac{\varphi\left(t_{k}\right) \bmod 2 \pi}{2 \pi}
$$

The phase coupling index $(\lambda)$ was defined by

$$
\lambda\left(t_{j}\right)=\left\|\frac{1}{N} \sum_{j=k-N}^{k} e^{-i \psi\left(t_{j}\right)}\right\|^{2}
$$

where $N$ is the number of heartbeats in time window of $t_{k}-t_{w} / 2 \leq t_{j}<t_{k}+t_{w} / 2$. $\lambda$ ranges from 0 to 1 , with $\lambda=1$ being the highest synchronization. $\psi\left(t_{k}\right)$ and respective $\lambda$ values were computed for different Fetal:Maternal $(n: m)$ heartbeat ratios, where $n$ and $m$ are fetal and maternal heartbeats, respectively. In this study, $N$ was set to 100 and $n: m$ coupling ratios associated with fetal beats of 1,2, and 3; and corresponding maternal beats of $n+2$ were investigated (i.e. the considered $n: m$ ratios are 1:3, 1:4, 2:4, 2:5, 3:5 and 3:6.

\subsection{Statistical Analysis}

The nonparametric Friedman test along with multiple comparison test were applied on the sample considered in this preliminary study to examine the significance in a two-way layout. Friedman tests the null hypothesis that the column effects are all the same against the alternative that they are not. Likewise, multiple comparison test performs multiple pairwise comparison of the group means (effects of anesthesia in this study). A p-value results from each test, which is informally a measure of the evidence against the null hypothesis (i.e. effects are not all the same). To determine the significance of the associations between anesthesia and HRV parameters, as well 
as anesthesia and coupling-based features, $\mathrm{p}<0.05$ was considered to be significant. Scheffe's procedure for multiple pairwise testing was used after $\mathrm{p}$-value was significant. The statistical analyses was applied on the three segments of the recorded ECG signals, i.e. P1 (0-5 min), P2 (5-10 min), and P3 (10-15 min).

\section{Results}

The preliminary study presented in this paper is conducted to explore the significant effects of anesthesia on fetal-maternal HR coupling patterns as well as fetal and maternal HRV parameters. Fig. 2 shows an example from a pregnant mouse and one of its fetuses including MHR, FHR, $\psi$ and $\lambda$ at ratio $1: 3$.
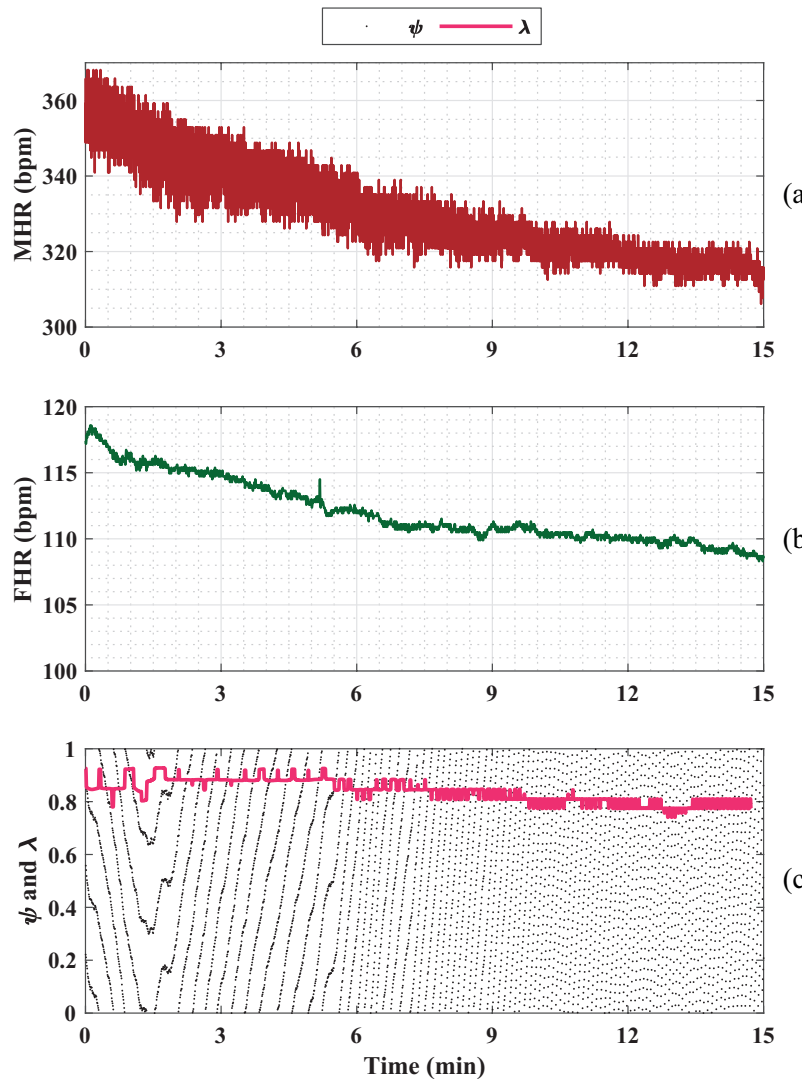

Figure 2. An example from a pregnant mouse and one of its fetuses, including: (a) maternal heart rate (MHR), (b) fetal heart rate (FHR), and (c) relative phase $(\psi)$ and coupling strength $(\lambda)$, both at a ratio of $1: 3$.

Table 1 lists mean \pm SD values for different maternal and fetal HRV parameters considering the three segments (P1 (0-5 min), P2 (5-10 min), and P3 (10-15 min)) of the recorded signals. Values highlighted in bold correspond to $\mathrm{p}<0.05$, i.e. the null hypothesis is rejected, when conducting the Friedman and multiple comparison tests. Results showed that MMHR and MRMSSDHR significantly $(\mathrm{p}<0.05)$ decreased from P1 to P3 $(324.46 \pm 17.18 \mathrm{bpm}(\mathrm{P} 1)$ to $296.31 \pm 20.47 \mathrm{bpm}(\mathrm{P} 3) ;$ and $12.24 \pm 8.59 \mathrm{bpm}(\mathrm{P} 1)$ to $6.64 \pm 5.28$ bpm (P3), respectively). Furthermore, FMHR, FSDNNHR and FRMSSDHR decreased from P1 to P3 (100.22 $\pm 13.16 \mathrm{bpm}(\mathrm{P} 1)$ to $89.38 \pm 10.53 \mathrm{bpm}(\mathrm{P} 3)$; $7.08 \pm 3.92 \mathrm{bpm}(\mathrm{P} 1)$ to $2.11 \pm 1.80 \mathrm{bpm}(\mathrm{P} 3)$; and $1.76 \pm 1.59$ $\mathrm{bpm}(\mathrm{P} 1)$ to $0.65 \pm 1.01 \mathrm{bpm}(\mathrm{P} 3)$, respectively). No significant changes were found between P1 to P2, or P2 to P3. Moreover, fetal-maternal $\lambda$ patterns did not change significantly among the three segments of the recorded signals.

Table 1. Descriptive statistics (mean \pm SD) for HRV parameters and coupling measures for different ratios showing the effect of anesthesia considering the three segments (P1, P2 and P3) of the recorded signals. Values highlighted in bold indicate significant difference (i.e. $\mathrm{p}<0.05$ ). MMHR or FMHR: Mean value of maternal or fetal heart rate. MSDNNHR or FSDNNHR: Standard deviation of NN intervals in maternal or fetal heart rate. MRMSSDHR or FRMSSDHR: Root mean square of successive differences between normal maternal or fetal heartbeats. NN intervals: Interbeat intervals from which artifacts have been removed.

\begin{tabular}{llll}
\hline \hline $\begin{array}{l}\text { Descriptive } \\
\text { Statistics }\end{array}$ & $\begin{array}{c}\text { P1 } \\
(0-5 \mathrm{~min})\end{array}$ & $\begin{array}{c}\text { P2 } \\
(5-10 \mathrm{~min})\end{array}$ & $\begin{array}{c}\text { P3 } \\
(10-15 \mathrm{~min})\end{array}$ \\
\hline $\begin{array}{l}\text { MMHR } \\
\text { (bpm) }\end{array}$ & $\mathbf{3 2 4} \pm \mathbf{1 7}$ & $308 \pm 19$ & $\mathbf{2 9 6} \pm \mathbf{2 0}$ \\
$\begin{array}{l}\text { MSDNNHR } \\
\text { (bpm) }\end{array}$ & $9.7 \pm 6.1$ & $7.0 \pm 2.9$ & $5.4 \pm 2.5$ \\
MRMSSDHR & $\mathbf{1 2 . 2} \pm \mathbf{8 . 6}$ & $8.1 \pm 6.3$ & $\mathbf{6 . 6} \pm \mathbf{5 . 3}$ \\
(bpm) & & & \\
FMHR (bpm) & $\mathbf{1 0 0} \pm \mathbf{1 3}$ & $93 \pm 11$ & $\mathbf{8 9} \pm \mathbf{1 1}$ \\
FSDNNHR & $\mathbf{7 . 1} \pm \mathbf{3 . 9}$ & $2.5 \pm 1.5$ & $\mathbf{2 . 1} \pm \mathbf{1 . 8}$ \\
(bpm) & & & \\
FRMSSDHR & $\mathbf{1 . 8} \pm \mathbf{1 . 6}$ & $0.9 \pm 1.0$ & $\mathbf{0 . 7} \pm \mathbf{1 . 0}$ \\
(bpm) & & \\
mean $(\lambda[1: 3])$ & $0.4 \pm 0.3$ & $0.5 \pm 0.3$ & $0.5 \pm 0.3$ \\
mean $(\lambda[1: 4])$ & $0.2 \pm 0.2$ & $0.2 \pm 0.2$ & $0.2 \pm 0.2$ \\
mean $(\lambda[2: 4])$ & $0 \pm 0$ & $0 \pm 0$ & $0 \pm 0$ \\
mean $(\lambda[2: 5])$ & $0.1 \pm 0.1$ & $0 \pm 0$ & $0 \pm 0.1$ \\
mean $(\lambda[3: 5])$ & $0 \pm 0$ & $0 \pm 0$ & $0 \pm 0$ \\
mean $(\lambda[3: 6])$ & $0 \pm 0$ & $0 \pm 0$ & $0 \pm 0$ \\
\hline \hline
\end{tabular}

\section{Discussion}

This paper presented a preliminary study to investigate the significant effects of anesthesia on fetal and maternal HRV features in addition to fetal-maternal HR cou- 
pling parameters at various ratios. It can be depicted from Fig. 2 that anesthesia injected into mother mouse caused a decrease in both of maternal and fetal HR, in general. In addition, fetal-maternal HR synchronization could have the possibility of placental transmission to fetal circulation [11] which could aid in the assessment of autonomic regulation of maternal and fetal heart and their interactions.

The conducted Friedman and multiple comparison tests showed that maternal and fetal HRV parameters including: MMHR and MRMSSDHR, as well as FMHR, FSDNNHR and FRMSSDHR significantly decreased from P1 to P3 (i.e. after the first $10 \mathrm{~min}$ ). On the other hand, no significant changes were observed for the coupling patterns between fetal-maternal heartbeats. This could potentially indicate the possibility of placental transmission of anesthesia to fetal circulation. These observations suggest that fetal sympathetic and parasympathetic nervous system activities were suppressed by anesthesia treatment applied to pregnant mice for more than $10 \mathrm{~min}$.

\section{Conclusions}

The changes in fetal and maternal HRV parameters in addition to fetal-maternal HR coupling measures during anesthesia were assessed in the preliminary study presented in this paper. This is essential when studying the cardiovascular system activities in the utero of pregnant mouse under anesthesia, which is compulsory for surgical procedure. The features of HRV and coupling measures were evaluated for three segments of the recorded ECG signals from 10 fetuses and 6 pregnant mice. Due to the small number of samples, the study requires further validation on a large sample size to obtain robust results from the statistical tests.

The changes in heartbeat synchronization patterns and HRV parameters were assessed using the Friedman and multiple comparison statistical tests. Results demonstrated that MMHR and MRMSSDHR, as well as FMHR, FSDNNHR and FRMSSDHR significantly decreased from $\mathrm{P} 1$ to $\mathrm{P} 3$, i.e. after the first $10 \mathrm{~min}$. No significant changes were observed between P1 to P2, or P2 to P3. Likewise, fetal-maternal heartbeats coupling patterns did not change significantly between the three segments. The examined results suggest that anesthesia treatment applied to pregnant mice for more than 10 min suppressed the activities of fetal sympathetic and parasympathetic nervous systems.

\section{Acknowledgments}

The study was partly supported by an internal grant awarded to AH Khandoker (CIRA 2019-023 grant Project 8474000174).

\section{References}

[1] Rossant J. Mouse mutants and cardiac development. Circ Res March 1996;78(3):349-353.

[2] Khandoker AH, Al Khoori T, Ito T, Minato T, Kimura Y. Effect of chronic hypoxia on autonomic nervous system of fetal mice. In 2017 Computing in Cardiology (CinC). Rennes, France, September 24-27, 2017; 1-4.

[3] Mu J, Slevin JC, Qu D, McCormick S, Adamson SL. In vivo quantification of embryonic and placental growth during gestation in mice using micro-ultrasound. Reprod Biol Endocrinol August 2008;6(1):34-46.

[4] Shetty A, Venkatesh T, Tsutsumi R, Suresh PS. Gene expression changes and promoter methylation with the combined effects of estradiol and leptin in uterine tissue of the ovariectomized mice model of menopause. Mol Biol Rep October 2020;47(1):151-168.

[5] Kaufman MH. The Atlas of Mouse Development. First edition. New York: Academic Press, July 1992.

[6] Task Force of the European Society of Cardiology the North American Society of Pacing Electrophysiology. Heart rate variability. Circulation March 1996;93(5):1043-1065.

[7] Khandoker AH, Al Khoori T, Ito T, Sugibayashi R, Kimura Y. Assessment of autonomic neurodevelopment in the mouse fetuses by using fetal electrocardiography. In 2016 38th Annual International Conference of the IEEE Engineering in Medicine and Biology Society (EMBC). Orlando, FL, USA, August 16-20, 2016; 2954-2957.

[8] He M, et al. Association between heart rate variability indices and features of spontaneous ventricular tachyarrhythmias in mice. Clin Exp Pharmacol Physiol February 2020; 47(7):1193-1202.

[9] Khandoker $\mathrm{AH}$, et al. Regulation of maternal-fetal heart rates and coupling in mice fetuses. In 2018 40th Annual International Conference of the IEEE Engineering in Medicine and Biology Society (EMBC). Honolulu, HI, USA, July 18-21, 2018; 5257-5260.

[10] Niizeki K, Saitoh T. Cardiolocomotor phase synchronization during rhythmic exercise. J Phys Fit Sports Med March 2014;3(1):11-20.

[11] Khandoker AH, et al. Effect of $\beta$-blocker on maternal-fetal heart rates and coupling in pregnant mice and fetuses. In 2019 41st Annual International Conference of the IEEE Engineering in Medicine and Biology Society (EMBC). Berlin, Germany, July 23-27, 2019; 1784-1787.

Address for correspondence:

Maisam Wahbah

Healthcare Engineering Innovation Center (HEIC), Department of Biomedical Engineering, Khalifa University, Abu Dhabi 127788, UAE

maisam.wahbah@ku.ac.ae and ahsan.khandoker@ku.ac.ae 